\title{
Differential inequalities imposed by the extended hypergeometric function
}

\author{
Rabha W. Ibrahim ${ }^{1 *}$, M. Z. Ahmad² and Hiba F. Al-Janaby²
}

\author{
*Correspondence: \\ rabhaibrahim@yahoo.com \\ ${ }^{1}$ Faculty of Computer \\ Science and Information \\ Technology, University \\ Malaya, 50603 Kuala Lumpur, \\ Malaysia \\ Full list of author information \\ is available at the end of the \\ article
}

\begin{abstract}
Recently, the generalized hypergeometric function is extended by utilizing the Beta function. Based on this type of function, we introduce a new operator in the open unit disk. The present article investigates some subordination and superordination results for certain normalized analytic functions in the open unit disk, which are acted upon by the generalized Noor integral operator. Some of outcomes improve and generalize previously known outcomes.
\end{abstract}

Keywords: Analytic function, Univalent function, Integral operator, Subordination, Superordination, Hypergeometric function, Unit disk

Mathematics Subject Classification: 30C45

\section{Background}

The theory of hypergeometric functions theaters significant and imposing role in the study of the fractional calculus and the geometric function theory. It motivates the theory of univalent functions, by attractive to current research after their utilization in the proof of great famous problem in geometric function theory which is called by the Bieberbach's conjecture. This theory has been developed with enriched many presentations and simplification by protruding complex analysis.

Let $H(\mathcal{U})$ be the class of all holomorphic functions $\phi(z)$ which are defined in the unit disk $\mathcal{U}$. For $\alpha \in \mathbb{C}$ and $n \in \mathbb{N}$, we let $H[a, n]=\left\{\phi \in H(\mathcal{U}): \phi(z)=a+a_{n} z^{n}+a_{n+1} z^{n+1}+\cdots\right\}$ and $\mathcal{A}$ be the subclass of $H(\mathcal{U})$ consisting of functions of the form

$$
\phi(z)=z+\sum_{n=2}^{\infty} a_{n} z^{n}, \quad(z \in \mathcal{U}) .
$$

For functions $\phi(z)$, given (1), and $\psi(z)$ given by

$$
\psi(z)=z+\sum_{n=2}^{\infty} b_{n} z^{n}, \quad(z \in \mathcal{U}) .
$$

the Hadamard product (or convolution) of $\phi(z)$ and $\psi(z)$ is defined by

$$
(\phi * \psi)(z)=z+\sum_{n=2}^{\infty} a_{n} b_{n} z^{n}, \quad(z \in \mathcal{U}) .
$$

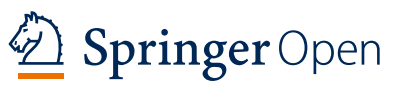

(c) 2016 Ibrahim et al. This article is distributed under the terms of the Creative Commons Attribution 4.0 International License (http://creativecommons.org/licenses/by/4.0/), which permits unrestricted use, distribution, and reproduction in any medium, provided you give appropriate credit to the original author(s) and the source, provide a link to the Creative Commons license, and indicate if changes were made. 
For $\phi$ and $\varphi$ be members of the function class $H(\mathcal{U})$, the function $\phi$ is said to be subordinate to $\varphi$, or $\varphi$ is superordinate to $\phi$, if there is an analytic function $\theta(z)$ in $\mathcal{U}$ with $\theta(0)=0$ and $|\theta(z)|<1$ for all $z \in \mathcal{U}$, such that $\phi(z)=\varphi(\theta(z))$. In this case, we write $\phi \prec \varphi$, or $\phi(z) \prec \varphi(z)$. Furthermore, if the function $\varphi$ is univalent in $\mathcal{U}$, then we have the following equivalence (Miller and Mocanu 2000):

$$
\phi(z) \prec \varphi(z) \quad(z \in \mathcal{U}) \Longleftrightarrow \phi(0)=\varphi(0), \quad \phi(\mathcal{U}) \subset \varphi(\mathcal{U})
$$

Let $\phi: \mathbb{C}^{2} \longrightarrow \mathbb{C}$ and let $\vartheta$ be univalent in $z \in \mathcal{U}$. If $\rho$ is analytic in $\mathcal{U}$ and satisfies the differential subordination $\phi\left(\rho(z), z \rho^{\prime}(z)\right) \prec \vartheta(z)$ then $\rho$ is called a solution of the differential subordination. The univalent function $\eta$ is called a dominant of the solutions of the differential subordination, $\rho \prec \eta$. If $\rho$ and $\phi\left(\rho(z), z \rho^{\prime}(z)\right)$ are univalent in $\mathcal{U}$ and satisfy the differential superordination $\vartheta(z) \prec \phi\left(\rho(z), z \rho^{\prime}(z)\right)$ then $\rho$ is called a solution of the differential superordination. An analytic $\eta$ is called subordinate of the solution of the differential superordination if $\eta \prec \rho$ (Miller and Mocanu 2003).

For real or complex numbers $\alpha, \beta, \gamma$ other than $0,-1,-2, \ldots$ the Gaussian hypergeometric function is defined by de Branges (1985)

$$
F(\alpha, \beta ; \gamma, z)=\sum_{n=0}^{\infty} \frac{(\alpha)_{n}(\beta)_{n}}{(\gamma)_{n}(1)_{n}} z^{n}=1+\frac{\alpha \beta}{\gamma} z+\frac{\alpha(\alpha+1) \beta(\beta+1)}{\gamma(\gamma+1)} \frac{z^{2}}{2 !}+\cdots
$$

where $(\alpha)_{n}$ is the Pochhammer symbol defined by

$$
(\alpha)_{n}=\frac{\Gamma(\alpha+n)}{\Gamma(\alpha)}= \begin{cases}1, & n=0 \\ \alpha(\alpha+1) \ldots(\alpha+n-1), & n=\{1,2, \ldots\}\end{cases}
$$

and achieved

$$
\begin{aligned}
& \text { (i) } n(\wp)_{n}=\wp\left[(\wp+1)_{n}-(\wp)_{n}\right] \\
& \text { (ii) }(\wp)_{n+1}=\wp(\wp+1)_{n}=(\wp+n)(\wp)_{n} .
\end{aligned}
$$

Let $B(x, y)$ be the familiar Beta function defined by Srivastava et al. (2012, p. 8))

$$
B(x, y)= \begin{cases}\int_{0}^{1} \sigma^{x-1}(1-\sigma)^{y-1} d \sigma & (\Re(x)>0 ; \Re(y)>0) \\ \frac{\Gamma(x) \Gamma(y)}{\Gamma(x+y)} & \left(x, y \in \mathbb{C}_{0}^{-}\right)\end{cases}
$$

Here $\Gamma$ denotes the Euler's Gamma function (see, e.g., Srivastava and Choi 2012, Section 1.1). Srivastava et al. (2014) $\mathcal{B}_{p}^{a, b ; \kappa, \mu}(x, y)$, introduced the extended Beta function as follows

$$
\begin{aligned}
& \mathcal{B}_{p}^{a, b ; \kappa, \mu}(x, y)=\int_{0}^{1} \sigma^{x-1}(1-\sigma)^{y-1} F\left(a ; b ;-\frac{p}{\sigma^{\kappa}(1-\sigma)^{\mu}}\right) d \sigma, \\
& \quad(\kappa \geq 0, \mu \geq 0, \mathfrak{R}(p) \geq 0, \min \{\mathfrak{R}(a), \mathfrak{R}(b)\}>0, \mathfrak{R}(x)>-\mathfrak{R}(\kappa a), \mathfrak{R}(y)>-\mathfrak{R}(\mu a)) .
\end{aligned}
$$

The special case of (5) when $p=0$ is seen to immediately reduce to the familiar beta function $B(x, y)(\min \{\Re(x), \Re(y)\}>0)$ (Srivastava and Choi 2012).

Agarwal et al. (2015) introduced the extended Gauss hypergeometric function as follows 


$$
\begin{gathered}
F_{p ; \kappa, \mu}(\alpha, \beta ; \gamma ; z ; m):=\sum_{n=0}^{\infty} \frac{(\alpha)_{n}(\beta)_{n}}{(\gamma)_{n}} \frac{B_{p}^{a, b ; \kappa, \mu}(\beta+n, \gamma-\beta+m)}{B(\beta+n, \gamma-\beta+m)} \frac{z^{n}}{n !} \\
(p \geq 0, \mathfrak{R}(\kappa)>0, \mathfrak{R}(\mu)>0, m<\mathfrak{R}(\beta)<\mathfrak{R}(\gamma),|z|<1) .
\end{gathered}
$$

The special case of (6) $p=0, m=0$ is noted to reduce to the ordinary Gauss hypergeometric function $F(\alpha, \beta ; \gamma ; z)$ (Agarwal et al. 2015). Ibrahim et al. (2015b) introduced a generalized Noor integral operator using a fractional hypergeometric function as follows:

$$
\begin{aligned}
& Q_{p ; \kappa, \mu}^{\wp}(\gamma ; \alpha, \beta ; z ; m): \mathcal{A} \longrightarrow \mathcal{A} \\
& Q_{p ; \kappa, \mu}^{\wp}(\gamma ; \alpha, \beta ; z ; m) \phi(z)=\Omega\left(z F_{p}^{a, b ; \kappa, \mu}(\alpha, \beta ; \gamma ; z ; m)\right)^{-1} * \phi(z), \quad(z \in \mathcal{U}) \\
& Q_{p ; \kappa, \mu}^{\wp}(\gamma ; \alpha, \beta ; z ; m) \phi(z) \\
& \quad=z+\sum_{n=2}^{\infty} \frac{(\gamma)_{n-1}}{(\alpha)_{n-1}(\beta)_{n-1}} \frac{\Omega B(\beta+n-1, \gamma-\beta+m)}{B_{p}^{a, b ; \kappa, \mu}(\beta+n-1, \gamma-\beta+m)}(\wp+1)_{n-1} a_{n} z^{n},
\end{aligned}
$$

where

$$
\begin{aligned}
& \Omega=\frac{B_{p}^{a, b ; \kappa, \mu}(\beta, \gamma-\beta+m)}{B(\beta, \gamma-\beta+m)}, \\
& \left(z F_{p}^{a, b ; \kappa, \mu}(\alpha, \beta ; \gamma ; z ; m)\right)^{-1} \\
& \quad=\sum_{n=1}^{\infty} \frac{(\gamma)_{n-1}}{(\alpha)_{n-1}(\beta)_{n-1}} \frac{B(\beta+n-1, \gamma-\beta+m)}{B_{p}^{a, b ; \kappa, \mu}(\beta+n-1, \gamma-\beta+m)}(\wp+1)_{n-1} z^{n} .
\end{aligned}
$$

In view of (7), we get

$$
\begin{aligned}
& z\left[Q_{p ; \kappa, \mu}^{\wp}(\gamma ; \alpha, \beta ; z ; m) \phi(z)\right]^{\prime} \\
& \quad=(\wp+1) Q_{p ; \kappa, \mu}^{\wp+1}(\gamma ; \alpha, \beta ; z ; m) \phi(z)-\wp Q_{p ; \kappa, \mu}^{\wp}(\gamma ; \alpha, \beta ; z ; m) \phi(z) .
\end{aligned}
$$

In particular, we have

$$
Q_{0 ; \kappa, \mu}^{\wp}(\gamma ; \alpha, \beta ; z ; 0) \phi(z)=I_{\wp}(\alpha, \beta, \gamma) \phi(z)
$$

where the integral operator $I_{\wp}(\alpha, \beta, \gamma) \phi(z)$ was investigate by Noor (2006).

Making use of the principle of subordination various subordination theorems involving certain operators for analytic functions in $\mathcal{U}$ were investigated by Miller and Mocanu (2000) and Owa and Srivastava (2004). Further, Bulboaca (2002a, b) and Miller and Mocanu (2003) extended the study to differential superordination as the dual problem of differential subordination, later the study has been taken by many researchers such as, Ali et al. (2005), Murugusundaramoorthy and Magesh (2006), and Shanmugam et al. (2006), Magesh and Murugusundaramoorthy (2008), Mostafa and Aouf (2009), Aouf and Mostafa (2010), Cho et al. (2011), Magesh (2011), Ibrahim et al. (2015a), and others. 
Related to the present investigation, we mention some of them in recent years. In Ibrahim and Darus (2008), the first author applied a method based on the differential subordination and superordination in order to obtain results involving generalized Noor integral operator utilizing the Fox- Wright function for a normalized analytic function $\phi(z), z \in \mathcal{U}$ and is denoted by $I_{\lambda}\left[\left(\alpha_{j}, A_{j}\right)_{1, q} ;\left(\beta_{j}, B_{j}\right)_{1, p}\right] \phi(z)$. Also they studied the sufficient condition to satisfy

$$
\eta_{1}(z) \prec \frac{\left[z I_{\lambda}\left[\left(\alpha_{j}, A_{j}\right)_{1, q} ;\left(\beta_{j}, B_{j}\right)_{1, p}\right] \phi(z)\right]^{\prime}}{\Phi\left[I_{\lambda}\left[\left(\alpha_{j}, A_{j}\right)_{1, q} ;\left(\beta_{j}, B_{j}\right)_{1, p}\right] \phi(z)\right]} \prec \eta_{2}(z)
$$

for some convex functions $\eta_{1}$ and $\eta_{2}$. with $\eta_{1}(0) \neq 0$ and $\eta_{2}(0) \neq 0$.

Aouf and Seoudy (2013) investigated some subordination and superordination results for certain p-valent functions in the open unit disc, which acted upon by a class of a linear operator denoted by $I_{p, q, s, \lambda}^{m, l}\left(\alpha_{1}\right) \phi(z)$. Further, they studied the sufficient condition to satisfy

$$
\eta_{1}(z) \prec\left[\frac{I_{p, q, s, \lambda}^{m, l}\left(\alpha_{1}\right) \phi(z)}{z^{p}}\right]^{\mu}\left[\frac{z^{p}}{I_{p, q, s, \lambda}^{m, l}\left(\alpha_{1}+1\right) \phi(z)}\right]^{v} \prec \eta_{2}(z),
$$

for some convex functions $\eta_{1}$ and $\eta_{2}$. with $\eta_{1}(0) \neq 0$ and $\eta_{2}(0) \neq 0$.

Magesh et al. (2014) studied the subordination and superordination results of the linear operator denoted by $\Theta\left[\alpha_{1}\right](\phi)(z)$. Also, they discussed the sufficient condition to satisfy

$$
\eta_{1}(z) \prec\left[\frac{\Theta\left[\alpha_{1}\right](\phi * \Phi)(z)}{z}\right]^{\mu}\left[\frac{z}{\Theta\left[\alpha_{1}+1\right](\phi * \Psi(z))}\right]^{v} \prec \eta_{2}(z),
$$

for some convex functions $\eta_{1}$ and $\eta_{2}$ with $\eta_{1}(0) \neq 0$ and $\eta_{2}(0) \neq 0$ and $\Phi(z)=z+\sum_{n=2}^{\infty} \lambda_{n} z^{n}, \Psi(z)=z+\sum_{n=2}^{\infty} \mu_{n} z^{n}$ are analytic functions in $\mathcal{U}$ with $\lambda_{n} \geq 0, \mu_{n} \geq 0$ and $\lambda_{n} \geq \mu_{n}$.

Ibrahim et al. (2015b) investigated some differential subordination and superordination results regarding the generalized integral operator defined by (7). Moreover, we investigate sufficient condition for a normalized analytic function $\phi(z), z \in \mathcal{U}$ to satisfy

$$
\eta_{1}(z) \prec \frac{z\left[Q_{p ; \kappa, \mu}^{\wp}(\gamma ; \alpha, \beta ; z ; m) \phi(z)\right]^{\prime}}{\Phi\left[Q_{p ; \kappa, \mu}^{\wp}(\gamma ; \alpha, \beta ; z ; m) \phi(z)\right]} \prec \eta_{2}(z),
$$

for some convex functions $\eta_{1}$ and $\eta_{2}$.

In this present paper, we study some differential subordination and superordination results for new subclasses regarding the generalized integral operator defined by (7). Moreover, we investigate sandwich results containing the given generalized integral operator for certain a normalized analytic function $\phi(z), z \in \mathcal{U}$ such that $(\phi * \Theta)(z) \neq 0$ to satisfy

$\eta_{1}(z) \prec\left[\frac{Q_{p ; \kappa, \mu}^{\wp}(\gamma ; \alpha, \beta ; z ; m)(\phi * \Phi)(z)}{z}\right]^{\sigma}\left[\frac{z}{Q_{p ; \kappa, \mu}^{\wp}(\gamma ; \alpha, \beta ; z ; m)(\phi * \Theta)(z)}\right]^{v} \prec \eta_{2}(z)$, 
for some convex functions $\eta_{1}$ and $\eta_{2}$ and $\Phi(z)=z+\sum_{n=2}^{\infty} \lambda_{n} z^{n}, \Psi(z)=z+\sum_{n=2}^{\infty} \mu_{n} z^{n}$ are analytic functions in $\mathcal{U}$ with $\lambda_{n} \geq 0, \mu_{n} \geq 0$ and $\lambda_{n} \geq \mu_{n}$.

\section{Preliminaries}

In order to prove our subordination and superordination results, we need to the following lemmas in the sequel.

Definition 1 (Miller and Mocanu 2003) Let $\mathcal{Q}$ denote the set of functions $\phi$ that are analytic and univalent on $\overline{\mathcal{U}} \backslash \mathcal{E}(\phi)$, where $\mathcal{E}(\phi)=\left\{\xi \in \partial \mathcal{U}: \lim _{z \longrightarrow \xi} \phi(z)=\infty\right\}$, is such that $\min \left|\phi^{\prime}(\xi)\right|=p>0$ for $\xi \in \partial \mathcal{U} \backslash \mathcal{E}(\phi)$.

Lemma 1 (Miller and Mocanu 2000) Let $\rho$ be univalent in the unit disk $\mathcal{U}$ and $\Psi$ and let $\Lambda$ be analytic in a domain $\mathcal{D}$ containing $\rho(\mathcal{U})$ with $\phi(\omega) \neq 0$ when $\omega \in \eta(\mathcal{U})$. Set $\mathcal{Q}(z):=z \eta^{\prime}(z) \Lambda(\eta(z))$ and $\vartheta(z):=\Psi(\rho(z))+\mathcal{Q}(z)$. Suppose that

1. $\mathcal{Q}(z)$ is starlike univalent in $\mathcal{U}$,

2. $\mathfrak{R}\left(z \vartheta^{\prime}(z) / \mathcal{Q}(z)\right)>0$ for $z \in \mathcal{U}$.

If $\rho$ is analytic with $\rho(0)=\eta(0), \rho(\mathcal{U}) \subset \mathcal{D}$ and

$\Psi(\rho(z))+z \rho^{\prime}(z) \Lambda(\rho(z)) \prec \Psi(\eta(z))+z \eta^{\prime}(z) \Lambda(\eta(z))$,

then $\rho(z) \prec \eta(z)$, and $\eta(z)$ is the best dominant.

Lemma 2 (Bulboaca 2002a) Let $\eta$ be convex univalent in the unit disk $\mathcal{U}$ and $\Pi$ and let $\Delta$ be analytic in a domain $\mathcal{D}$ containing $\eta(\mathcal{U})$. Suppose that

1. $z \eta^{\prime}(z) \Delta(\eta(z))$ is starlike univalent in $\mathcal{U}$,

2. $\left.\mathfrak{R}\left\{\Pi^{\prime}(\eta(z)) / \Delta(\eta(z))\right\}\right)>0$ for $z \in \mathcal{U}$.

If $\rho(z) \in H[\eta(0), 1] \cap \mathcal{Q}$ with $\rho(\mathcal{U}) \subseteq \mathcal{D}$ and $\Pi(\rho(z))+z \rho^{\prime}(z) \Delta(\rho(z))$ being univalent in $\mathcal{U}$ and

$$
\begin{aligned}
& \Pi(\eta(z))+z \eta^{\prime}(z) \Delta(\eta(z)) \prec \Pi(\rho(z))+z \rho^{\prime}(z) \Delta(\rho(z)), \\
& \text { then } \eta(z) \prec \rho(z), \text { and } \eta(z) \text { is the best subordinant. }
\end{aligned}
$$

\section{Sandwich outcomes}

By making use of Lemmas 1 , we first prove the following subordination results.

Theorem 1 Let $\Phi, \Psi \in \mathcal{A}, \lambda_{i} \in \mathbb{C}(i=1,2,3), \lambda_{3} \neq 0, \sigma, v \in \mathbb{C}$ and $\eta(z) \neq 0$ be univalent in $\mathcal{U}$ such that $z \eta^{\prime}(z) / \eta(z)$ is starlike univalent in $\mathcal{U}$ and

$$
\mathfrak{R}\left\{1+\frac{\lambda_{2}}{\lambda_{3}} \eta(z)+\frac{z \eta^{\prime \prime}(z)}{\eta^{\prime}(z)}-\frac{z \eta^{\prime}(z)}{\eta(z)}\right\}>0, \quad(z \in \mathcal{U}) .
$$

If $\phi \in \mathcal{A}$ satisfies the subordination 


$$
\begin{aligned}
\lambda_{1} & +\lambda_{2}\left[\frac{Q_{p ; \kappa, \mu}^{\wp}(\gamma ; \alpha, \beta ; z ; m)(\phi * \Phi)(z)}{z}\right]^{\sigma}\left[\frac{z}{Q_{p ; \kappa, \mu}^{\wp}(\gamma ; \alpha, \beta ; z ; m)(\phi * \Theta)(z)}\right]^{v} \\
& +\lambda_{3} \sigma(\wp+1)\left[\frac{Q_{p ; \kappa, \mu}^{\wp+1}(\gamma ; \alpha, \beta ; z ; m)(\phi * \Phi)(z)}{Q_{p ; \kappa, \mu}^{\wp}(\gamma ; \alpha, \beta ; z ; m)(\phi * \Phi)(z)}-1\right] \\
& +\nu(\wp+2)\left[1-\frac{Q_{p ; \kappa, \mu}^{\wp+2}(\gamma ; \alpha, \beta ; z ; m)(\phi * \Theta)(z)}{Q_{p ; \kappa, \mu}^{\wp+1}(\gamma ; \alpha, \beta ; z ; m)(\phi * \Theta)(z)}\right] \\
& \prec \lambda_{1}+\lambda_{2} \eta(z)+\lambda_{3} \frac{z \eta^{\prime}(z)}{\eta(z)}
\end{aligned}
$$

then

$$
\left[\frac{Q_{p ; \kappa, \mu}^{\wp}(\gamma ; \alpha, \beta ; z ; m)(\phi * \Phi)(z)}{z}\right]^{\sigma}\left[\frac{z}{Q_{p ; \kappa, \mu}^{\wp}(\gamma ; \alpha, \beta ; z ; m)(\phi * \Theta)(z)}\right]^{\nu} \prec \eta(z),
$$

and $\eta(z)$ is the best dominant.

Proof Our aim is to apply Lemma 1. Setting

$$
\rho(z)=\left[\frac{Q_{p ; \kappa, \mu}^{\wp}(\gamma ; \alpha, \beta ; z ; m)(\phi * \Phi)(z)}{z}\right]^{\sigma}\left[\frac{z}{Q_{p ; \kappa, \mu}^{\wp}(\gamma ; \alpha, \beta ; z ; m)(\phi * \Theta)(z)}\right]^{\nu} .
$$

Computation shows that

$$
\begin{aligned}
\frac{z \rho^{\prime}(z)}{\rho(z)}= & \lambda_{3} \sigma(\wp+1)\left[\frac{Q_{p ; \kappa, \mu}^{\wp+1}(\gamma ; \alpha, \beta ; z ; m)(\phi * \Phi)(z)}{Q_{p ; \kappa, \mu}^{\wp}(\gamma ; \alpha, \beta ; z ; m)(\phi * \Phi)(z)}-1\right] \\
& +v(\wp+2)\left[1-\frac{Q_{p ; \kappa, \mu}^{\wp+2}(\gamma ; \alpha, \beta ; z ; m)(\phi * \Theta)(z)}{Q_{p ; \kappa, \mu}^{\wp+1}(\gamma ; \alpha, \beta ; z ; m)(\phi * \Theta)(z)}\right],
\end{aligned}
$$

which yields the following subordination:

$$
\lambda_{1}+\lambda_{2} \rho(z)+\lambda_{3} \frac{z \rho^{\prime}(z)}{\rho(z)} \prec \lambda_{1}+\lambda_{2} \eta(z)+\lambda_{3} \frac{z \eta^{\prime}(z)}{\eta(z)} .
$$

By setting

$$
\Psi(\omega):=\lambda_{1}+\lambda_{2} \omega, \quad \Lambda(\omega):=\frac{\lambda_{3}}{\omega}, \pi \neq 0,
$$

it can be easily observed that $\Psi(\omega)$ is analytic in $\mathbb{C}$ and $\Lambda(\omega)$ is analytic in $\mathbb{C} \backslash\{0\}$ and that $\Lambda(\omega) \neq 0$ when $\omega \in \mathbb{C} \backslash\{0\}$. Also, by letting

$$
\begin{aligned}
& \mathcal{Q}(z)=z \eta^{\prime}(z) \Lambda(\eta(z))=\lambda_{3} \frac{z \eta^{\prime}(z)}{\eta(z)}, \\
& \vartheta(z)=\Psi(\eta(z))+\mathcal{Q}(z)=\lambda_{1}+\lambda_{2} \eta(z)+\lambda_{3} \frac{z \eta^{\prime}(z)}{\eta(z)},
\end{aligned}
$$


we find that $\mathcal{Q}(z)$ is starlike univalent in $\mathcal{U}$ and that

$$
\mathfrak{R}\left\{\frac{z \vartheta^{\prime}(z)}{\mathcal{Q}(z)}\right\}=\mathfrak{R}\left\{1+\frac{\lambda_{2}}{\lambda_{3}} \eta(z)+\frac{z \eta^{\prime \prime}(z)}{\eta^{\prime}(z)}-\frac{z \eta^{\prime}(z)}{\eta(z)}\right\}>0 .
$$

Corollary 1 Let the assumptions of Theorem 1 hold. Then the subordination

$$
\begin{aligned}
\lambda_{1} & +\lambda_{2}\left[\frac{Q_{p ; \kappa, \mu}^{\wp}(\gamma ; \alpha, \beta ; z ; m)(\phi * \Phi)(z)}{z}\right]^{\sigma}+\lambda_{3} \sigma(\wp+1)\left[\frac{Q_{p ; \kappa, \mu}^{\wp+1}(\gamma ; \alpha, \beta ; z ; m)(\phi * \Phi)(z)}{Q_{p ; \kappa, \mu}^{\wp}(\gamma ; \alpha, \beta ; z ; m)(\phi * \Phi)(z)}-1\right] \\
& +\prec \lambda_{1}+\lambda_{2} \eta(z)+\lambda_{3} \frac{z \eta^{\prime}(z)}{\eta(z)}
\end{aligned}
$$

implies

$$
\left[\frac{Q_{p ; \kappa, \mu}^{\wp}(\gamma ; \alpha, \beta ; z ; m)(\phi * \Phi)(z)}{z}\right]^{\sigma} \prec \eta(z),
$$

and $\eta(z)$ is the best dominant.

Proof By letting $v=0$ in Theorem 1, we have the required result.

Corollary 2 Let the assumptions of Theorem 1 hold. Then the subordination

$$
\begin{aligned}
\lambda_{1} & +\lambda_{2}\left[\frac{z}{Q_{p ; \kappa, \mu}^{\wp}(\gamma ; \alpha, \beta ; z ; m)(\phi * \Theta)(z)}\right]^{v}+v(\wp+2)\left[1-\frac{Q_{p ; \kappa, \mu}^{\wp+2}(\gamma ; \alpha, \beta ; z ; m)(\phi * \Theta)(z)}{Q_{p ; \kappa, \mu}^{\wp+1}(\gamma ; \alpha, \beta ; z ; m)(\phi * \Theta)(z)}\right] \\
& \prec \lambda_{1}+\lambda_{2} \eta(z)+\lambda_{3} \frac{z \eta^{\prime}(z)}{\eta(z)},
\end{aligned}
$$

implies

$$
\left[\frac{z}{Q_{p ; \kappa, \mu}^{\wp}(\gamma ; \alpha, \beta ; z ; m)(\phi * \Theta)(z)}\right]^{v} \prec \eta(z),
$$

and $\eta(z)$ is the best dominant.

Proof By letting $\sigma=0$ in Theorem 1, we have the required result.

Theorem 2 Let $\eta(z) \neq 0$ be convex univalent in the open unit disk $\mathcal{U}$. Suppose that

$$
\mathfrak{R}\left\{\frac{\lambda_{2}}{\lambda_{3}} \eta(z)\right\} \geq 0, \quad v, \pi \in \mathbb{C}, \pi \neq 0, \text { for } z \in \mathcal{U},
$$

and that $z \eta^{\prime}(z) / \eta(z)$ is starlike univalent in $\mathcal{U}$.

$$
\text { If }\left[\frac{Q_{p ; \kappa, \mu}^{\wp}(\gamma ; \alpha, \beta ; z ; m)(\phi * \Phi)(z)}{z}\right]^{\sigma}\left[\frac{z}{Q_{p ; \kappa, \mu}^{\wp}(\gamma ; \alpha, \beta ; z ; m)(\phi * \Theta)(z)}\right]^{\nu} \in H[1,1] \cap \mathcal{Q} \text {, where } \Phi, \Theta \in \mathcal{A} \text {, }
$$




$$
\begin{aligned}
& \lambda_{1}+\lambda_{2}\left[\frac{Q_{p ; \kappa, \mu}^{\wp}(\gamma ; \alpha, \beta ; z ; m)(\phi * \Phi)(z)}{z}\right]^{\sigma}\left[\frac{z}{Q_{p ; \kappa, \mu}^{\wp}(\gamma ; \alpha, \beta ; z ; m)(\phi * \Theta)(z)}\right]^{v} \\
& +\lambda_{3} \sigma(\wp+1)\left[\frac{Q_{p ; \kappa, \mu}^{\wp+1}(\gamma ; \alpha, \beta ; z ; m)(\phi * \Phi)(z)}{Q_{p ; \kappa, \mu}^{\wp}(\gamma ; \alpha, \beta ; z ; m)(\phi * \Phi)(z)}-1\right] \\
& +v(\wp+2)\left[1-\frac{Q_{p ; \kappa, \mu}^{\wp+2}(\gamma ; \alpha, \beta ; z ; m)(\phi * \Theta)(z)}{Q_{p ; \kappa, \mu}^{\wp+1}(\gamma ; \alpha, \beta ; z ; m)(\phi * \Theta)(z)}\right],
\end{aligned}
$$

is univalent in $\mathcal{U}$ and the subordination

$$
\begin{aligned}
\lambda_{1} & +\lambda_{2} \eta(z)+\lambda_{3} \frac{z \eta^{\prime}(z)}{\eta(z)} \\
& \prec \lambda_{1}+\lambda_{2}\left[\frac{Q_{p ; \kappa, \mu}^{\wp}(\gamma ; \alpha, \beta ; z ; m)(\phi * \Phi)(z)}{z}\right]^{\sigma}\left[\frac{z}{Q_{p ; \kappa, \mu}^{\wp}(\gamma ; \alpha, \beta ; z ; m)(\phi * \Theta)(z)}\right]^{v} \\
& +\lambda_{3} \sigma(\wp+1)\left[\frac{Q_{p ; \kappa, \mu}^{\wp+1}(\gamma ; \alpha, \beta ; z ; m)(\phi * \Phi)(z)}{Q_{p ; \kappa, \mu}^{\wp ;}(\gamma ; \alpha, \beta ; z ; m)(\phi * \Phi)(z)}-1\right] \\
& +v(\wp+2)\left[1-\frac{Q_{p ; \kappa, \mu}^{\wp+2}(\gamma ; \alpha, \beta ; z ; m)(\phi * \Theta)(z)}{Q_{p ; \kappa, \mu}^{\wp+1}(\gamma ; \alpha, \beta ; z ; m)(\phi * \Theta)(z)}\right]
\end{aligned}
$$

holds, then

$$
\eta(z) \prec\left[\frac{Q_{p ; \kappa, \mu}^{\wp}(\gamma ; \alpha, \beta ; z ; m)(\phi * \Phi)(z)}{z}\right]^{\sigma}\left[\frac{z}{Q_{p ; \kappa, \mu}^{\wp}(\gamma ; \alpha, \beta ; z ; m)(\phi * \Theta)(z)}\right]^{\nu},
$$

and $\eta(z)$ is the best subordinant.

Proof Our aim is to apply Lemma 2. Setting

$$
\rho(z)=\left[\frac{Q_{p ; \kappa, \mu}^{\wp}(\gamma ; \alpha, \beta ; z ; m)(\phi * \Phi)(z)}{z}\right]^{\sigma}\left[\frac{z}{Q_{p ; \kappa, \mu}^{\wp}(\gamma ; \alpha, \beta ; z ; m)(\phi * \Theta)(z)}\right]^{\nu} .
$$

Computation shows that

$$
\begin{aligned}
\frac{z \rho^{\prime}(z)}{\rho(z)}= & \lambda_{3} \sigma(\wp+1)\left[\frac{Q_{p ; \kappa, \mu}^{\wp+1}(\gamma ; \alpha, \beta ; z ; m)(\phi * \Phi)(z)}{Q_{p ; \kappa, \mu}^{\wp}(\gamma ; \alpha, \beta ; z ; m)(\phi * \Phi)(z)}-1\right] \\
& +v(\wp+2)\left[1-\frac{Q_{p ; \kappa, \mu}^{\wp+2}(\gamma ; \alpha, \beta ; z ; m)(\phi * \Theta)(z)}{Q_{p ; \kappa, \mu}^{\wp+1}(\gamma ; \alpha, \beta ; z ; m)(\phi * \Theta)(z)}\right],
\end{aligned}
$$

which yields the following subordination:

$$
\lambda_{1}+\lambda_{2} \eta(z)+\lambda_{3} \frac{z \eta^{\prime}(z)}{\eta(z)} \prec \lambda_{1}+\lambda_{2} \rho(z)+\lambda_{3} \frac{z \rho^{\prime}(z)}{\rho(z)} .
$$


By setting

$$
\Pi(\omega):=\lambda_{1}+\lambda+2 \omega, \quad \Delta(\omega):=\frac{\lambda_{3}}{\omega}, \pi \neq 0,
$$

it can be easily observed that $\Pi(\omega)$ is analytic in $\mathbb{C}$ and $\Delta(\omega)$ is analytic in $\mathbb{C} \backslash\{0\}$ and that $\Delta(\omega) \neq 0$ when $\omega \in \mathbb{C} \backslash\{0\}$. Also, we obtain

$$
\mathfrak{R}\left\{\frac{\Pi^{\prime}(\eta(z))}{\Delta(\eta(z))}\right\}=\Re\left\{\frac{\lambda_{2}}{\lambda_{3}} \eta(z)\right\}>0 .
$$

Then the relation (12) follows by an application of Lemma 2 .

Corollary 1 Let the assumptions of Theorem 1 hold. Then the subordination

$$
\begin{aligned}
\lambda_{1} & +\lambda_{2}\left[\frac{Q_{p ; \kappa, \mu}^{\wp}(\gamma ; \alpha, \beta ; z ; m)(\phi * \Phi)(z)}{z}\right]^{\sigma} \\
& +\lambda_{3} \sigma(\wp+1)\left[\frac{Q_{p ; \kappa, \mu}^{\wp+1}(\gamma ; \alpha, \beta ; z ; m)(\phi * \Phi)(z)}{Q_{p ; \kappa, \mu}^{\wp}(\gamma ; \alpha, \beta ; z ; m)(\phi * \Phi)(z)}-1\right] \\
& +\prec \lambda_{1}+\lambda_{2} \eta(z)+\lambda_{3} \frac{z \eta^{\prime}(z)}{\eta(z)},
\end{aligned}
$$

implies

$$
\eta(z) \prec\left[\frac{Q_{p ; \kappa, \mu}^{\wp}(\gamma ; \alpha, \beta ; z ; m)(\phi * \Phi)(z)}{z}\right]^{\sigma},
$$

and $\eta(z)$ is the best subordinant.

Proof By letting $v=0$ in Theorem 1, we have the required result.

Corollary 3 Let the assumptions of Theorem 1 hold. Then the subordination

$$
\begin{aligned}
\lambda_{1} & +\lambda_{2}\left[\frac{z}{Q_{p ; \kappa, \mu}^{\wp}(\gamma ; \alpha, \beta ; z ; m)(\phi * \Theta)(z)}\right]^{v} \\
& +v(\wp+2)\left[1-\frac{Q_{p ; \kappa, \mu}^{\wp+2}(\gamma ; \alpha, \beta ; z ; m)(\phi * \Theta)(z)}{Q_{p ; \kappa, \mu}^{\wp+1}(\gamma ; \alpha, \beta ; z ; m)(\phi * \Theta)(z)}\right] \\
& \prec \lambda_{1}+\lambda_{2} \eta(z)+\lambda_{3} \frac{z \eta^{\prime}(z)}{\eta(z)},
\end{aligned}
$$

implies

$$
\eta(z) \prec\left[\frac{z}{Q_{p ; \kappa, \mu}^{\wp}(\gamma ; \alpha, \beta ; z ; m)(\phi * \Theta)(z)}\right]^{v},
$$

and $\eta(z)$ is the best subordinant.

Proof By letting $\sigma=0$ in Theorem 1, we have the required result. 
Combining Theorems 1 and 2, in order to get the following sandwich theorem.

Theorem 3 Let $\eta_{1}(z) \neq 0, \eta_{2}(z) \neq 0$ be convex univalent in the open unit disk $\mathcal{U}, \lambda_{i} \in \mathbb{C}(i=1,2,3), \lambda_{3} \neq 0, \sigma, v \in \mathbb{C}$ and let $\eta_{2}(z)$ satisfy (9) and $\eta_{1}(z)$ satisfy (11) respectively. Suppose that and that $z \eta_{i}^{\prime}(z) / \eta_{i}(z), i=1,2$, is starlike univalent in $\mathcal{U}$. If

$$
\begin{gathered}
{\left[\frac{Q_{p ; \kappa, \mu}^{\wp}(\gamma ; \alpha, \beta ; z ; m)(\phi * \Phi)(z)}{z}\right]^{\sigma}\left[\frac{z}{Q_{p ; \kappa, \mu}^{\wp}(\gamma ; \alpha, \beta ; z ; m)(\phi * \Theta)(z)}\right]^{v} \in H[1,1] \cap \mathcal{Q}, \text { where } \Phi, \Theta \in \mathcal{A},} \\
\lambda_{1}+\lambda_{2}\left[\frac{Q_{p ; \kappa, \mu}^{\wp}(\gamma ; \alpha, \beta ; z ; m)(\phi * \Phi)(z)}{z}\right]^{\sigma}\left[\frac{z}{Q_{p ; \kappa, \mu}^{\wp}(\gamma ; \alpha, \beta ; z ; m)(\phi * \Theta)(z)}\right]^{v} \\
+\lambda_{3} \sigma(\wp+1)\left[\frac{Q_{p ; \kappa, \mu}^{\wp+1}(\gamma ; \alpha, \beta ; z ; m)(\phi * \Phi)(z)}{Q_{p ; \kappa, \mu}^{\wp}(\gamma ; \alpha, \beta ; z ; m)(\phi * \Phi)(z)}-1\right] \\
+v(\wp+2)\left[1-\frac{Q_{p ; \kappa, \mu}^{\wp+2}(\gamma ; \alpha, \beta ; z ; m)(\phi * \Theta)(z)}{Q_{p ; \kappa, \mu}^{\wp+1}(\gamma ; \alpha, \beta ; z ; m)(\phi * \Theta)(z)}\right],
\end{gathered}
$$

is univalent in $\mathcal{U}$ and the subordination

$$
\begin{aligned}
\lambda_{1} & +\lambda_{2} \eta_{1}(z)+\lambda_{3} \frac{z \eta_{1}^{\prime}(z)}{\eta_{1}(z)} \\
& \prec \lambda_{1}+\lambda_{2}\left[\frac{Q_{p ; \kappa, \mu}^{\wp}(\gamma ; \alpha, \beta ; z ; m)(\phi * \Phi)(z)}{z}\right]^{\sigma}\left[\frac{z}{Q_{p ; \kappa, \mu}^{\wp}(\gamma ; \alpha, \beta ; z ; m)(\phi * \Theta)(z)}\right]^{v} \\
& +\lambda_{3} \sigma(\wp+1)\left[\frac{Q_{p ; \kappa, \mu}^{\wp+1}(\gamma ; \alpha, \beta ; z ; m)(\phi * \Phi)(z)}{Q_{p ; \kappa, \mu}^{\wp}(\gamma ; \alpha, \beta ; z ; m)(\phi * \Phi)(z)}-1\right] \\
& +v(\wp+2)\left[1-\frac{Q_{p ; \kappa, \mu}^{\wp+2}(\gamma ; \alpha, \beta ; z ; m)(\phi * \Theta)(z)}{Q_{p ; \kappa, \mu}^{\wp+1}(\gamma ; \alpha, \beta ; z ; m)(\phi * \Theta)(z)}\right] \\
& \prec \lambda_{1}+\lambda_{2} \eta_{2}(z)+\lambda_{3} \frac{z \eta_{2}^{\prime}(z)}{\eta_{2}(z)},
\end{aligned}
$$

holds, then

$\eta_{1}(z) \prec\left[\frac{Q_{p ; \kappa, \mu}^{\wp}(\gamma ; \alpha, \beta ; z ; m)(\phi * \Phi)(z)}{z}\right]^{\sigma}\left[\frac{z}{Q_{p ; \kappa, \mu}^{\wp}(\gamma ; \alpha, \beta ; z ; m)(\phi * \Theta)(z)}\right]^{v} \prec \eta_{2}(z)$,

and $\eta_{1}(z)$ is the best subordinant and $\eta_{2}(z)$ is the best dominant.

Corollary 4 Let the assumptions of Theorem 3 hold and satisfy the subordination 


$$
\begin{aligned}
\lambda_{1} & +\lambda_{2} \frac{1+A_{1} z}{1+B_{1} z}+\lambda_{3} \frac{\left(A_{1}-B_{1}\right) z}{\left(1+A_{1} z\right)\left(1+B_{1} z\right)} \\
& \prec \lambda_{1}+\lambda_{2}\left[\frac{Q_{p ; \kappa, \mu}^{\wp}(\gamma ; \alpha, \beta ; z ; m)(\phi * \Phi)(z)}{z}\right]^{\sigma}\left[\frac{z}{Q_{p ; \kappa, \mu}^{\wp}(\gamma ; \alpha, \beta ; z ; m)(\phi * \Theta)(z)}\right]^{v} \\
& +\lambda_{3} \sigma(\wp+1)\left[\frac{Q_{p ; \kappa, \mu}^{\wp+1}(\gamma ; \alpha, \beta ; z ; m)(\phi * \Phi)(z)}{Q_{p ; \kappa, \mu}^{\wp}(\gamma ; \alpha, \beta ; z ; m)(\phi * \Phi)(z)}-1\right] \\
& +v(\wp+2)\left[1-\frac{Q_{p ; \kappa, \mu}^{\wp+2}(\gamma ; \alpha, \beta ; z ; m)(\phi * \Theta)(z)}{Q_{p ; \kappa, \mu}^{\wp+1}(\gamma ; \alpha, \beta ; z ; m)(\phi * \Theta)(z)}\right] \\
& \prec \lambda_{1}+\lambda_{2} \frac{1+A_{2} z}{1+B_{2} z}+\lambda_{3} \frac{\left(A_{2}-B_{2}\right) z}{\left(1+A_{2} z\right)\left(1+B_{2} z\right)},
\end{aligned}
$$

Then

$$
\begin{aligned}
\frac{1+A_{1} z}{1+B_{1} z} & \prec\left[\frac{Q_{p ; \kappa, \mu}^{\wp}(\gamma ; \alpha, \beta ; z ; m)(\phi * \Phi)(z)}{z}\right]^{\sigma}\left[\frac{z}{Q_{p ; \kappa, \mu}^{\wp}(\gamma ; \alpha, \beta ; z ; m)(\phi * \Theta)(z)}\right]^{v} \\
& \prec \frac{1+A_{2} z}{1+B_{2} z}
\end{aligned}
$$

and $\frac{1+A_{1} z}{1+B_{1} z}$ is the best subordinant and $\frac{1+A_{2} z}{1+B_{2} z}$ is the best dominant.

Proof Setting $\quad \eta_{1}(z)=\frac{1+A_{1} z}{1+B_{1} z}\left(-1 \leq B_{1}<A_{1} \leq 1\right) \quad$ and $\quad \eta_{2}(z)=\frac{1+A_{2} z}{1+B_{2} z}\left(-1 \leq B_{2}\right.$ $<A_{2} \leq 1$ ) in Theorem 3 .

\section{Conclusion}

By the term of the extend fractional hypergeometric function, we defined a new fractional integral operator in the open unit disk. This operator is a generalization of the well known Noor integral operator. Based on this operator, many subclasses may introduce in the geometric function theory. In this study, we concerned with a specific class of analytic function called of convolution type. This class imposed several well known classes.

Authors' contributions

RWI, MZA and HFA jointly worked on deriving the results. All authors read and approved the final manuscript.

\section{Author details}

${ }^{1}$ Faculty of Computer Science and Information Technology, University Malaya, 50603 Kuala Lumpur, Malaysia. ${ }^{2}$ Institute of Engineering Mathematics, Universiti Malaysia Perlis, 02600 Arau, Malaysia.

\section{Competing interests}

The authors declare that they have no competing interests.

Received: 15 September 2015 Accepted: 14 March 2016

Published online: 25 March 2016

\section{References}

Agarwal P, Choi J, Paris RB (2015) Extended Riemann-Liouville fractional derivative operator and its applications. J Nonlinear Sci Appl 8:451-466

Ali R, Ravichandran V, Khan MH, Subramanian G (2005) Differential sandwich theorems for certain analytic functions. Far East J Math Sci 15:87-94

Aouf MK, Mostafa AO (2010) Theorems for analytic functions defined by convolution. Acta Univ Apulensis Math Inform $21: 7-20$ 
Aouf MK, Seoudy TM (2013) Differential sandwich theorems of $p$-valent analtic functions involving a linear operator. An Şt Univ Ovidius Constanţa 21(1):5-18

Bulboaca T (2002a) A class of superordination-preserving integral operators. Indag Math (NS) 13:301-311

Bulboaca T (2002b) Classes of first-order differential superordinations. Demonstr Math 35(2):287-292

Cho NE, Kwon OS, Ali R, Ravichandaran V (2011) Subordination and superordination for multivalent functions associated with the Dziok-Srivastava operator. J Inequal Appl. Article ID 486595

de Branges L (1985) A proof of the Bieberbach conjecture. Acta Math 154(1-2):137-152

Ibrahim RW, Ahmad MZ, Al-Janaby HF (2015a) Third-order differential subordination and superordination involving a fractional operator. Open Math 13(1):706-728. doi:10.1515/math-2015-0068

Ibrahim RW, Ahmad MZ, Al-Janaby HF (2015b) Upper and lower bounds of integral operator defined by the fractional hypergeometric function. Open Math 13(1):768-780. doi:10.1515/math-2015-0071

Ibrahim RW, Darus M (2008) New classes of analytic functions involving generalized Noor integral operator. J Inequal Appl. Article ID 390435, pp 1-14

Magesh N (2011) Differential sandwich results for certain subclasses of analytic functions. Math Comput Model 54:803-814

Magesh N, Jothibasu J, Murthy S (2014) Subordination and superordination results associated with the generalized hypergeometric function. Math Slovaca 64(5):1197-1216

Magesh N, Murugusundaramoorthy G (2008) Differential subordinations and superordinations for a comprehensive class of analytic functions. SUT J Math 44:237-255

Miller SS, Mocanu PT (2000) Differential subordinations, theory and applications, monographs and textbooks in pure and applied mathematics, vol 225. Dekker, New York

Miller SS, Mocanu PT (2003) Subordinants of differetial superordinations. Complex Var Theory Appl 48(10):815-826

Mostafa AO, Aouf MK (2009) Sandwich theorems for certain subclasses of analytic functions defined by family of linear operators. J Appl Anal 15:269-280

Murugusundaramoorthy G, Magesh N (2006) Differential subordinations and superordinations for analytic functions defined by the Dziok-Srivastava linear operator. J Inequal Pure Appl Math 7:1-9

Noor KL (2006) Integral operators defined by convolution with hypergeometric functions. Appl Math Comput 182:1872-1881

Owa S, Srivastava HM (2004) Some subordination theorems involving a certain family of integral operators. Integral Transform Spec Funct 15:445-454

Shanmugam TN, Ravichandran V, Sivasubramanian S (2006) Differential sandwich theorems for some subclasses of analytic functions. Aust J Math Anal Appl 3(1):1-11

Srivastava HM, Agarwal P, Jain S (2014) Generating functions for the generalized Gauss hypergeometric functions. Appl Math Comput 247:348-352

Srivastava HM, Choi J (2012) Zeta and q-zeta functions and associated series and integrals. Elsevier, Amsterdam

\section{Submit your manuscript to a SpringerOpen ${ }^{\circ}$ journal and benefit from:}

- Convenient online submission

- Rigorous peer review

- Immediate publication on acceptance

- Open access: articles freely available online

- High visibility within the field

- Retaining the copyright to your article

Submit your next manuscript at $\mathbf{s p r i n g e r o p e n . c o m ~}$ 